Homology, Homotopy and Applications, vol.9(1), 2007, pp.213-220

\title{
CLASSIFICATION OF DI-EMBEDDINGS OF THE $n$-CUBE INTO $\mathbb{R}^{n}$
}

\author{
PRAPHAT FERNANDES AND ANDREW NICAS
}

(communicated by Gunnar Carlsson)

\begin{abstract}
A di-embedding of the $n$-cube $I^{n}$ into $\mathbb{R}^{n}$ is a map $I^{n} \rightarrow \mathbb{R}^{n}$ which is a dihomeomorphism onto its image. We show that such a map is, up to a permutation of coordinates, an $n$-fold product of 1-dimensional orientation preserving embeddings $I^{1} \rightarrow \mathbb{R}$.
\end{abstract}

\section{Introduction}

Geometric models for concurrent computing called progress graphs first appeared in $[\mathbf{2}]$ where they were attributed to Dijkstra. Progress graphs were used in [1] to prove the existence of deadlocks in certain types of concurrent systems. More recently, progress graphs appear in the work of Fajstrup, Goubault, Raussen and others $[\mathbf{3}, \mathbf{5}, \mathbf{6}]$ where various tools from algebraic topology are adapted to their study. One works in the category of directed spaces, also known as locally partially ordered spaces (abbreviated here as "LPO-spaces"), where execution paths in a concurrent system correspond to dipaths ("directed paths") in an LPO-space. In this language, progress graphs are partially ordered spaces whose partial order is induced by the Euclidean product partial order. An important problem is to determine when two execution paths give the same result; in [3], it is argued that this is the case when the corresponding dipaths are dihomotopic, i.e., homotopic through a family of dipaths. The fundamental category of an LPO-space, analogous to the classical fundamental groupoid, is constructed in [3]. Dihomeomorphism is the categorical notion of isomorphism in the category of LPO-spaces, and the fundamental category is a dihomeomorphism invariant. Various categories of fractions of the fundamental category, as well as their corresponding component categories are studied in [7]. The reader is referred to $[\mathbf{3}, \mathbf{5}]$ for a treatment of ditopology ("directed topology").

In this paper we show that the notion of dihomeomorphism is rather restrictive. A di-embedding of the $n$-cube $I^{n}=\prod_{i=1}^{n}[0,1]$ into $\mathbb{R}^{n}$ is a map $I^{n} \rightarrow \mathbb{R}^{n}$ which is a dihomeomorphism onto its image. Given a permutation $\sigma$ of $\{1, \ldots, n\}$, the induced coordinate permutation $\sigma_{*}: \mathbb{R}^{n} \rightarrow \mathbb{R}^{n}$ is the dihomeomorphism given by $\sigma_{*}\left(x_{1}, \ldots, x_{n}\right)=\left(x_{\sigma(1)}, \ldots, x_{\sigma(n)}\right)$. We prove (see Theorem 2.5):

The second author was partially supported by a grant from the Natural Sciences and Engineering Research Council of Canada.

Received January 8, 2006, revised October 23, 2006; published on February 8, 2007.

2000 Mathematics Subject Classification: 68Q85, 55D99, 55P99.

Key words and phrases: Dihomeomorphism, partially ordered space, progress graph.

Copyright (C) 2007, International Press. Permission to copy for private use granted. 
Theorem. Let $f: I^{n} \rightarrow \mathbb{R}^{n}$ be a di-embedding. Then there exists a coordinate permutation $\sigma_{*}: \mathbb{R}^{n} \rightarrow \mathbb{R}^{n}$ and orientation preserving embeddings $q_{i}: I^{1} \rightarrow \mathbb{R}$ such that $f=\sigma_{*}\left(\prod_{i=1}^{n} q_{i}\right)$.

This theorem reinforces the general belief that a workable notion of equivalence between LPO-spaces which is weaker than dihomeomorphism would be useful.

As a corollary, we determine the structure of the group $\operatorname{DiHomeo}\left(I^{n}\right)$ of dihomeomorphisms of $I^{n}$ (see Corollary 2.10):

Corollary. There is an isomorphism of topological groups:

$$
\left.\operatorname{DiHomeo}\left(I^{n}\right) \cong \operatorname{(Homeo}^{+}\left(I^{1}\right)\right)^{n} \rtimes \Sigma_{n} \quad \text { (semidirect product) }
$$

where the symmetric group $\Sigma_{n}$ of permutations of $\{1,2, \ldots, n\}$ acts on $\left(\text { Homeo }^{+}\left(I^{1}\right)\right)^{n}$, the $n$-fold product of the group of orientation preserving homeomorphisms of the unit interval, by permuting factors.

\section{Ditopology}

For the convenience of the reader and to establish some basic terminology, we recall some basic definitions concerning topological spaces with a compatible partial order, $[\mathbf{3}, \mathbf{5}]$.

Given a set $X$ with a partial order denoted by " $\leqslant$ ", the associated partial order relation is the set $R=\{(x, y) \in X \times X \mid x \leqslant y\}$.

Definition 1.1. A PO-space ("partially ordered space") is a pair $(X, \leqslant)$ consisting of a topological space $X$ and a partial order $\leqslant$ whose associated partial order relation is closed as a subset of $X \times X$ with the product topology.

A subset $Z \subset X$ of a PO-space $(X, \leqslant)$ inherits a PO-space structure by giving $Z$ the subspace topology and by restricting the partial order to $Z$.

Example 1.2. The real line $\mathbb{R}^{1}$ is a PO-space with the usual topology and the usual partial order (given by $x \leqslant y$ if and only if $y-x$ is non-negative). For a positive integer $n$, let $\pi_{i}: \mathbb{R}^{n} \rightarrow \mathbb{R}, i=1, \ldots, n$, denote projection to the $i$-th factor. The (Euclidean) product partial order on $\mathbb{R}^{n}$ is defined coordinatewise, i.e., for $x, y \in \mathbb{R}^{n}$, $x \leqslant y$ if and only $\pi_{j}(x) \leqslant \pi_{j}(y)$ for $j=1, \ldots, n$. The partial order relation, $R \subset$ $\mathbb{R}^{n} \times \mathbb{R}^{n}$, for this partial order is given by $R=\bigcap_{j=1}^{n}\left\{(x, y) \in \mathbb{R}^{n} \times \mathbb{R}^{n} \mid \pi_{j}(x) \leqslant\right.$ $\left.\pi_{j}(y)\right\}$ which is a closed subset of $\mathbb{R}^{n} \times \mathbb{R}^{n}$ since each $\left\{(x, y) \in \mathbb{R}^{n} \times \mathbb{R}^{n} \mid \pi_{j}(x) \leqslant\right.$ $\left.\pi_{j}(y)\right\}$ is clearly closed. Hence $\mathbb{R}^{n}$ with the usual topology and the product partial order is a PO-space.

Definition 1.3. A dimap $f:(X, \leqslant) \rightarrow(Y, \leqslant)$ between two PO-spaces is a continuous map $f: X \rightarrow Y$ which preserves partial order, i.e. for all $u, v \in X, u \leqslant v$ implies $f(u) \leqslant f(v)$.

The category of PO-spaces is the category whose objects are PO-spaces and whose morphisms are dimaps (categorical composition is composition of functions). Isomorphisms in this category are called dihomeomorphisms, i.e., 
Definition 1.4. A dimap $f: X \rightarrow Y$ is a dihomeomorphism if there is a dimap $g: Y \rightarrow X$ such that $g f$ is the identity of $X$ and $f g$ is the identity of $Y$.

A dimap of PO-spaces which is a homeomorphism of underlying topological spaces need not be a dihomeomorphism, as in the following example.

Example 1.5. Let $I^{2}=[0,1] \times[0,1] \subset \mathbb{R}^{2}$ and $X=\left\{(x, y) \in \mathbb{R}^{2} \mid 0 \leqslant y \leqslant 1, y \leqslant x\right.$ $\leqslant y+1\}$ with PO-space structures inherited from product partial order on $\mathbb{R}^{2}$. The map $f: I^{2} \rightarrow X$ defined by $f(x, y)=(x+y, y)$ is a homeomorphism with inverse $g: X \rightarrow I^{2}$ given by $g(x, y)=(x-y, y)$. While $f$ is a dimap, $g$ is not a dimap.

Definition 1.6. A map $f: X \rightarrow Y$ between PO-spaces is a di-embedding if $f$ is a dihomeomorphism onto its image where $f(X)$ inherits its $\mathrm{PO}$-space structure from $Y$.

Remark 1.7. A continuous map $q:[a, b] \rightarrow \mathbb{R}$, where $a<b$, is injective if and only if it is strictly monotone. Hence any orientation preserving embedding $q:[a, b] \rightarrow \mathbb{R}$ is a di-embedding.

Let $X$ be a topological space. The topological boundary of $A \subset X$, denoted by $\operatorname{bd}(A)$, is $\operatorname{bd}(A)=\bar{A} \cap \overline{A^{c}}$ where $A^{c}$ denotes the complement of $A$ in $X$ and for $C \subset X, \bar{C}$ denotes the closure of $C$ in $X$. For a subspace $Y$ of $X$ and $A \subset X$, let $\operatorname{bd}_{Y}(Y \cap A)$ denote the topological boundary of $Y \cap A$ in the subspace $Y$.

We record the following elementary lemma for use in Lemma 2.3.

Lemma 1.8. Let $X$ be a topological space, $Y$ a subspace of $X$ and $A \subset X$. Then $\operatorname{bd}_{Y}(Y \cap A) \subset \operatorname{bd}_{X}(A)$.

Proof. Observe that $y \in \operatorname{bd}_{Y}(Y \cap A)$ if and only if for every neighborhood $V_{y}$ of $y$ in $X$ we have $Y \cap V_{y} \cap A \neq \emptyset$ and $Y \cap V_{y} \cap A^{c} \neq \emptyset$ (where $A^{c}$ is the complement of $A$ in $X$ ). These conditions imply that $V_{y} \cap A \neq \emptyset$ and $V_{y} \cap A^{c} \neq \emptyset$ and thus $y \in \operatorname{bd}_{X}(A)$.

\section{Di-embeddings of standard $n$-rectangles into $\mathbb{R}^{n}$}

In this section we prove the main theorem of this paper (Theorem 2.5) and derive some consequences (Corollaries 2.7, 2.9, 2.10, 2.11).

Let $R \subset \mathbb{R}^{n} \times \mathbb{R}^{n}$ be the partial order relation for the product partial order on $\mathbb{R}^{n}$ (see Example 1.2). Recall that $\pi_{i}: \mathbb{R}^{n} \rightarrow \mathbb{R}$ denotes projection to the $i$-th factor.

Lemma 2.1. The topological boundary of $R$ in $\mathbb{R}^{n} \times \mathbb{R}^{n}$ is given by

$$
\operatorname{bd}(R)=\bigcup_{j=1}^{n}\left\{(x, y) \in R \mid \pi_{j}(x)=\pi_{j}(y)\right\}
$$


Proof. The complement of $R$ in $\mathbb{R}^{n} \times \mathbb{R}^{n}$ is given by

$$
R^{c}=\bigcup_{j=1}^{n}\left\{(x, y) \in \mathbb{R}^{n} \times \mathbb{R}^{n} \mid \pi_{j}(x)>\pi_{j}(y)\right\}
$$

The closure of $R^{c}$ is given by:

$$
\overline{R^{c}}=\bigcup_{j=1}^{n} \overline{\left\{(x, y) \mid \pi_{j}(x)>\pi_{j}(y)\right\}}=\bigcup_{j=1}^{n}\left\{(x, y) \mid \pi_{j}(x) \geqslant \pi_{j}(y)\right\} .
$$

Hence

$$
\begin{gathered}
\operatorname{bd}(R)=\bar{R} \cap \overline{R^{c}}=R \cap \overline{R^{c}}= \\
\left(\bigcap_{j=1}^{n}\left\{(x, y) \mid \pi_{j}(x) \leqslant \pi_{j}(y)\right\}\right) \cap\left(\bigcup_{j=1}^{n}\left\{(x, y) \mid \pi_{j}(x) \geqslant \pi_{j}(y)\right\}\right) \\
=\bigcup_{j=1}^{n}\left\{(x, y) \in R \mid \pi_{j}(x)=\pi_{j}(y)\right\}
\end{gathered}
$$

For subset $X$ of $\mathbb{R}^{n}$, write $\left.R\right|_{X}=R \cap(X \times X)$. Note that $\left.R\right|_{X}$ is the partial order relation for the restriction of the product partial order on $\mathbb{R}^{n}$ to $X$.

A standard $n$-rectangle is a subset $P$ of $\mathbb{R}^{n}$ of the form $P=\prod_{i=1}^{n}\left[a_{i}, b_{i}\right]$ where each $\left[a_{i}, b_{i}\right]$ is a closed interval with $a_{i}<b_{i}$. A straightforward adaptation of the proof of Lemma 2.2 yields:

Lemma 2.2. Let $P$ be a standard n-rectangle. The topological boundary of $R$ in $P \times P$ is given by

$$
\operatorname{bd}_{P \times P}\left(\left.R\right|_{P}\right)=\bigcup_{j=1}^{n}\left\{\left.(x, y) \in R\right|_{P} \mid \pi_{j}(x)=\pi_{j}(y)\right\} .
$$

For $1 \leqslant j \leqslant n$ and $c \in \mathbb{R}$, define $H_{c}^{j}=\pi_{j}^{-1}(\{c\})$, a hyperplane perpendicular to the $j$-th coordinate axis. For a subset $A \subset \mathbb{R}^{n}$, we write $H_{c}^{j}(A)$ for $H_{c}^{j} \cap A$. Note that $H_{c}^{j} \cap H_{d}^{j} \neq \emptyset$ if and only if $c=d$. Also observe that if $j \neq k$ then $H_{c}^{j}(P) \cap H_{d}^{k}(P) \neq$ $\emptyset$ where $P=\prod_{i=1}^{n}\left[a_{i}, b_{i}\right]$ is a standard $n$-rectangle and $c \in\left[a_{j}, b_{j}\right]$ and $d \in\left[a_{k}, b_{k}\right]$.

Lemma 2.3. Let $f: P \rightarrow \mathbb{R}^{n}$ be a di-embedding of a standard $n$-rectangle $P=$ $\prod_{i=1}^{n}\left[a_{i}, b_{i}\right]$. Then for each index $1 \leqslant j \leqslant n$ and $c \in\left[a_{j}, b_{j}\right]$ there exists an index $1 \leqslant j(c) \leqslant n$ and a real number $q(c)$ such that $f\left(H_{c}^{j}(P)\right) \subset H_{q(c)}^{j(c)}$.

Proof. Let $S=f(P)$. By hypothesis, $f: P \rightarrow S$ is a dihomeomorphism. In particular, $f: P \rightarrow S$ is a homeomorphism and so $f \times f: P \times P \rightarrow S \times S$ is also a homeomorphism. Let $R \subset \mathbb{R}^{n} \times \mathbb{R}^{n}$ be the partial order relation for the product partial order on $\mathbb{R}^{n}$ (as in Lemma 2.1). Since $f: P \rightarrow S$ is a dihomeomorphism, for all $x, y \in P, f(x) \leqslant f(y)$ if and only if $x \leqslant y$ and hence $\left.(f \times f)\left(\left.R\right|_{P}\right) \subset R\right|_{S}$ and $\left.(f \times f)^{-1}\left(\left.R\right|_{S}\right) \subset R\right|_{P}$. It follows that $(f \times f)\left(\left.R\right|_{P}\right)=\left.R\right|_{S}$ because $f \times f$ is a 
bijection. Since $f \times f: P \times P \rightarrow S \times S$ is homeomorphism,

$$
(f \times f)\left(\operatorname{bd}_{P \times P}\left(\left.R\right|_{P}\right)\right)=\operatorname{bd}_{S \times S}\left(\left.R\right|_{S}\right) .
$$

Given $c \in\left[a_{j}, b_{j}\right]$, define points $a_{c}^{j}, b_{c}^{j} \in H_{c}^{j}(P)$ by $\pi_{j}\left(a_{c}^{j}\right)=\pi_{j}\left(b_{c}^{j}\right)=c$ and $\pi_{i}\left(a_{c}^{j}\right)=a_{i}, \pi_{i}\left(b_{c}^{j}\right)=b_{i}$ for $i \neq j$. Observe that $H_{c}^{j}(P)=\left\{x \in P \mid a_{c}^{j} \leqslant x \leqslant b_{c}^{j}\right\}$. By Lemma 2.2, $\left(a_{c}^{j}, b_{c}^{j}\right) \in \operatorname{bd}_{P \times P}\left(\left.R\right|_{P}\right)$ and so (1) implies that $\left(f\left(a_{c}^{j}\right), f\left(b_{c}^{j}\right)\right) \in$ $\operatorname{bd}_{S \times S}\left(\left.R\right|_{S}\right)$. By Lemma 1.8, $\operatorname{bd}_{S \times S}\left(\left.R\right|_{S}\right) \subset \operatorname{bd}(R)$ and so Lemma 2.1 implies there exists an index $j(c)$ such that $\pi_{j(c)}\left(f\left(a_{c}^{j}\right)\right)=\pi_{j(c)}\left(f\left(b_{c}^{j}\right)\right)$; denote this real number by $q(c)$. Suppose $x \in H_{c}^{j}(P)$. Then $a_{c}^{j} \leqslant x \leqslant b_{c}^{j}$ and so $f\left(a_{c}^{j}\right) \leqslant f(x) \leqslant f\left(b_{c}^{j}\right)$ since $f$ is a dimap. It follows that $\pi_{j(c)}\left(f\left(a_{c}^{j}\right)\right) \leqslant \pi_{j(c)}(f(x)) \leqslant \pi_{j(c)}\left(f\left(b_{c}^{j}\right)\right)$ and thus $\pi_{j(c)}(f(x))=q(c)$, i.e., $f(x) \in H_{q(c)}^{j(c)}$.

For a positive integer $n$, denote $\underline{n}=\{1, \ldots, n\}$. Given a permutation $\sigma$ of $\underline{n}$, i.e., a bijection $\sigma: \underline{n} \rightarrow \underline{n}$, the induced coordinate permutation, $\sigma_{*}: \mathbb{R}^{n} \rightarrow \mathbb{R}^{n}$, is the map given by $\pi_{j}\left(\sigma_{*}(x)\right)=\pi_{\sigma(j)}(x)$ for $x \in \mathbb{R}^{n}$. Note that $\sigma_{*}$ is a dihomeomorphism with inverse $\left(\sigma^{-1}\right)_{*}$.

Lemma 2.4. Let $P=\prod_{i=1}^{k}\left[a_{i}, b_{i}\right]$ be a standard $k$-rectangle, where $k \geqslant 1$, and let $u: P \rightarrow \mathbb{R}^{k}$ be a map of the form $u=\tau_{*}\left(\prod_{j=1}^{k} g_{j}\right)$, where $\tau_{*}: \mathbb{R}^{k} \rightarrow \mathbb{R}^{k}$ is a coordinate permutation and each $g_{j}:\left[a_{j}, b_{j}\right] \rightarrow \mathbb{R}$ is an embedding, $j=1, \ldots, k$. Let $v_{i}(P) \in P, \quad i=0,1, \ldots, k$, be the vertices $v_{0}(P)=\left(a_{1}, \ldots, a_{k}\right)$ and $v_{i}(P)=$ $\left(a_{1}, \ldots, a_{i-1}, b_{i}, a_{i+1}, \ldots, a_{k}\right), i=1, \ldots, k$. Then the vectors $u\left(v_{i}(P)\right)-u\left(v_{0}(P)\right)$, $i=1, \ldots, k$, are non-zero and mutually orthogonal.

Proof. Since the coordinate permutation $\tau_{*}: \mathbb{R}^{k} \rightarrow \mathbb{R}^{k}$ is an orthogonal linear transformation, it suffices to consider the case $\tau$ is the identity. Note that $u\left(v_{i}(P)\right)-$ $u\left(v_{0}(P)\right)=\left(g_{i}\left(b_{i}\right)-g_{i}\left(a_{i}\right)\right) e_{i}$, where $e_{i}$ is the $i$-th standard unit basis vector for $\mathbb{R}^{k}$, is not zero because $g_{i}$ is injective. The conclusion follows from the orthogonality of the standard basis.

Theorem 2.5. Let $f: P \rightarrow \mathbb{R}^{n}$ be a di-embedding of a standard $n$-rectangle $P=$ $\prod_{i=1}^{n}\left[a_{i}, b_{i}\right]$. Then there exists a coordinate permutation $\sigma_{*}: \mathbb{R}^{n} \rightarrow \mathbb{R}^{n}$ and orientation preserving embeddings $q_{i}:\left[a_{i}, b_{i}\right] \rightarrow \mathbb{R}$ such that $f=\sigma_{*}\left(\prod_{i=1}^{n} q_{i}\right)$.

Proof. The proof proceeds by induction on $n$. The case $n=1$ follows from Remark 1.7. Inductively, assume that the conclusion of the theorem is valid for di-embeddings of standard $(n-1)$-rectangles into $\mathbb{R}^{n-1}$. We will need the following strengthened version of Lemma 2.3:

Claim 2.6. For each integer $1 \leqslant j \leqslant n$, there exists $1 \leqslant k \leqslant n$ such that for all $c \in\left[a_{j}, b_{j}\right]$ there exists a real number $q(c)$ such that $f\left(H_{c}^{j}(P)\right) \subset H_{q(c)}^{k}$.

Proof of the Claim. By Lemma 2.3 , given an integer $1 \leqslant j \leqslant n$ and $c \in\left[a_{j}, b_{j}\right]$ there exists an integer $1 \leqslant k(c) \leqslant n$ and a real number $q(c)$ such that $f\left(H_{c}^{j}(P)\right) \subset H_{q(c)}^{k(c)}$. Let $\phi_{d}^{\ell}: H_{d}^{\ell} \rightarrow \mathbb{R}^{n-1}$ denote the canonical dihomeomorphism given by

$$
\phi_{d}^{\ell}\left(x_{1}, \ldots, x_{\ell-1}, d, x_{\ell+1}, \ldots, x_{n}\right)=\left(x_{1}, \ldots, x_{\ell-1}, x_{\ell+1}, \ldots, x_{n}\right) .
$$

Define $P_{j}=\prod_{i \neq j}\left[a_{i}, b_{i}\right]$ (a standard $(n-1)$-rectangle). Note that $\phi_{c}^{j}$ restricts to 
a dihomeomorphism $H_{c}^{j}(P) \rightarrow P_{j}$ which we also denote by $\phi_{c}^{j}$. By the inductive hypothesis applied to $\phi_{q(c)}^{k(c)} f\left(\phi_{c}^{j}\right)^{-1}$ and Lemma 2.4, the $n-1$ vectors $w_{i}(c)=$ $f\left(\left(\phi_{c}^{j}\right)^{-1}\left(v_{i}\left(P_{j}\right)\right)\right)-f\left(\left(\phi_{c}^{j}\right)^{-1}\left(v_{0}\left(P_{j}\right)\right)\right), i=1, \ldots, n-1$ (see Lemma 2.4 for the notation $\left.v_{i}\left(P_{j}\right)\right)$ are non-zero and orthogonal. Hence the cross product ${ }^{1}$ $\mathrm{CP}\left(w_{1}(c), \ldots, w_{n-1}(c)\right)$ is a vector in $\mathbb{R}^{n}$ which is non-zero and orthogonal to the hyperplane $H_{q(c)}^{k(c)}$. The set of unit vectors which are orthogonal to some hyperplane of the form $H_{d}^{\ell}$ is the finite set $S=\left\{ \pm e_{j} \mid j=1, \ldots, n\right\}$ where $e_{1}, \ldots, e_{n}$ is the standard basis for $\mathbb{R}^{n}$. The function $c \mapsto \mathrm{CP}\left(w_{1}(c), \ldots, w_{n-1}(c)\right) / \| \mathrm{CP}\left(w_{1}(c), \ldots\right.$, $\left.w_{n-1}(c)\right) \|$, where $\|v\|$ denotes the length of $v$, is a continuous function with domain $\left[a_{j}, b_{j}\right]$ taking values in the discrete set $S$, hence it is a constant and so $k(c)$ is independent of $c$, proving the claim.

In Claim 2.6, the integer $k$ is uniquely determined by $j$. In order to verify this assertion, suppose that for $c \in\left[a_{j}, b_{j}\right], f\left(H_{c}^{j}(P)\right) \subset H_{q(c)}^{k}$ and $f\left(H_{c}^{j}(P)\right) \subset H_{r(c)}^{\ell}$. It follows that $f\left(H_{a_{j}}^{j}(P)\right) \subset H_{q\left(a_{j}\right)}^{k} \cap H_{r\left(a_{j}\right)}^{\ell}$. If $k \neq \ell$ then $H_{q\left(a_{j}\right)}^{k} \cap H_{r\left(a_{j}\right)}^{\ell} \cong \mathbb{R}^{n-2}$

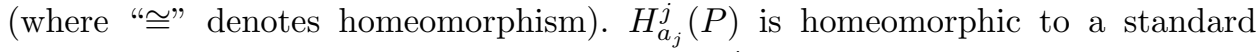
$(n-1)$-rectangle and the restriction of $f$ to $H_{a_{j}}^{j}(P)$ thus yields an embedding of a standard $(n-1)$-rectangle into $\mathbb{R}^{n-2}$ contradicting Invariance of Domain ${ }^{2}$. Note that the function $c \mapsto q(c)$ with the property $f\left(H_{c}^{j}(P)\right) \subset H_{q(c)}^{k}$ for every $c \in\left[a_{j}, b_{j}\right]$ is uniquely determined by $j$ and $f$. Suppose $c \mapsto p(c)$ is another function with the property $f\left(H_{c}^{j}(P)\right) \subset H_{p(c)}^{k}$. Then $H_{q(c)}^{k} \cap H_{p(c)}^{k}$ is not empty since it contains $f\left(H_{c}^{j}(P)\right)$ and so $q(c)=p(c)$. We will use the notation $q_{j}(c)$ for $q(c)$ to indicate its dependence on $j$.

Claim 2.6 yields a function $\sigma: \underline{n} \rightarrow \underline{n}$, where $\sigma(j)$ is the unique integer for which $f\left(H_{c}^{j}(P)\right) \subset H_{q_{j}(c)}^{\sigma(j)}$ for all $c \in\left[a_{j}, b_{j}\right]$. We show that $\sigma$ is injective. Suppose $\sigma(j)=\sigma(k)$. Then $f\left(H_{c}^{j}(P)\right) \subset H_{q_{j}(c)}^{\sigma(j)}$ for all $c \in\left[a_{j}, b_{j}\right]$ and $f\left(H_{d}^{k}(P)\right) \subset H_{q_{k}(d)}^{\sigma(k)}$ for all $d \in\left[a_{k}, b_{k}\right]$. If $j \neq k$ then $H_{c}^{j}(P) \cap H_{d}^{k}(P) \neq \emptyset$ and thus $H_{q_{j}(c)}^{\sigma(j)} \cap H_{q_{k}(d)}^{\sigma(k)} \neq \emptyset$. Since $\sigma(j)=\sigma(k)$, it follows that $q_{j}(c)=q_{k}(d)$ for all for all $c \in\left[a_{j}, b_{j}\right]$ and $d \in$ $\left[a_{k}, b_{k}\right]$. In particular, $q_{j}(c)$ is independent of $c$ and so $f\left(H_{c}^{j}(P)\right) \subset H_{q_{j}\left(a_{j}\right)}^{\sigma(j)}$ for all $c \in\left[a_{j}, b_{j}\right]$. Since $P=\cup_{c} H_{c}^{j}(P)$, it follows that $f(P) \subset H_{q_{j}\left(a_{j}\right)}^{\sigma(j)}$ yielding an embedding of the standard $n$-rectangle $P$ into $H_{q_{j}\left(a_{j}\right)}^{\sigma(j)} \cong \mathbb{R}^{n-1}$, contradicting Invariance of Domain. Thus $\sigma$ is injective and hence bijective, i.e. a permutation, since $\underline{n}$ is finite. Define $h: P \rightarrow \mathbb{R}^{n}$ by $h=\left(\sigma^{-1}\right)_{*} f$. Then $h\left(H_{c}^{j}(P)\right) \subset H_{q_{j}(c)}^{j}$ for all $c \in$ $\left[a_{j}, b_{j}\right]$ and $1 \leqslant j \leqslant n$. This condition is equivalent to $\pi_{j} h=q_{j}\left(\left.\pi_{j}\right|_{P}\right)$ for $1 \leqslant j \leqslant n$ where $\left.\pi_{j}\right|_{P}: P \rightarrow\left[a_{j}, b_{j}\right]$ is projection. Hence each $q_{j}:\left[a_{j}, b_{j}\right] \rightarrow \mathbb{R}$ is continuous

${ }^{1}$ For $k \geqslant 2$, the cross product of a $(k-1)$-tuple of vectors $w_{1}, \ldots, w_{k-1}$ in $\mathbb{R}^{k}$, denoted $\mathrm{CP}\left(w_{1}, \ldots, w_{k-1}\right)$, is the vector $\sum_{j=1}^{k}(-1)^{j} \operatorname{det}\left(A_{j}\right) e_{j}$, where $e_{1}, \ldots, e_{k}$ is the standard basis for $\mathbb{R}^{k}$ and $\operatorname{det}\left(A_{j}\right)$ is the determinant of the $(k-1) \times(k-1)$ matrix $A_{j}$ obtained from the $(k-1) \times k$ matrix $A$ whose $i$-th row is the vector $w_{i}$ by deleting the $j$-th column. If $w_{1}, \ldots, w_{k-1}$ in $\mathbb{R}^{k}$ are linearly independent, then $\mathrm{CP}\left(w_{1}, \ldots, w_{k-1}\right)$ is non-zero and orthogonal to each $w_{i}$.

${ }^{2}$ Invariance of Domain is the assertion that a subset of $\mathbb{R}^{n}$ which is homeomorphic to an open subset of $\mathbb{R}^{n}$ is itself an open subset of $\mathbb{R}^{n}$; see Theorem 16 in Section 4.7 of [8] 
and $h=\prod_{j=1}^{n} q_{j}$. Since $h$ is an embedding and a dimap, so is each $q_{j}$; furthermore, by Remark 1.7 each $q_{j}$ is an orientation-preserving embedding. We conclude $f=\sigma_{*}\left(\prod_{j=1}^{n} q_{j}\right)$.

The special case $n=2$ of Theorem 2.5 was proved in [4].

Corollary 2.7. Let $f: P \rightarrow \mathbb{R}^{n}$ be a di-embedding of a standard $n$-rectangle $P=$ $\prod_{i=1}^{n}\left[a_{i}, b_{i}\right]$. Then $f(P)$ is a standard n-rectangle.

Proof. The conclusion of the corollary is clear for a map of the form $f=$ $\sigma_{*}\left(\prod_{j=1}^{n} q_{j}\right)$ as in the conclusion of Theorem 2.5.

Remark 2.8. The proof of Theorem 2.5 shows that the di-embedding $f: P \rightarrow \mathbb{R}^{n}$ uniquely determines the permutation $\sigma$ and the orientation-preserving embeddings $q_{j}:\left[a_{j}, b_{j}\right] \rightarrow \mathbb{R}, j=1, \ldots, n$, such that $f=\sigma_{*}\left(\prod_{j=1}^{n} q_{j}\right)$.

A standard open $n$-rectangle is a subset $P$ of $\mathbb{R}^{n}$ of the form $P=\prod_{i=1}^{n}\left(a_{i}, b_{i}\right)$ where each $\left(a_{i}, b_{i}\right)$ is an open interval with $a_{i}<b_{i}$.

Corollary 2.9. Let $f: P \rightarrow \mathbb{R}^{n}$ be a di-embedding of a standard open $n$-rectangle $P=\prod_{i=1}^{n}\left(a_{i}, b_{i}\right)$. Then there exists a coordinate permutation $\sigma_{*}: \mathbb{R}^{n} \rightarrow \mathbb{R}^{n}$ and orientation-preserving embeddings $q_{i}:\left(a_{i}, b_{i}\right) \rightarrow \mathbb{R}$ such that $f=\sigma_{*}\left(\prod_{i=1}^{n} q_{i}\right)$.

Proof. Let $\delta=\frac{1}{3} \min \left\{\left|b_{i}-a_{i}\right| \mid i=1, \ldots, n\right\}$ and let $P_{m}=\prod_{i=1}^{n}\left[a_{i}+\delta / m, b_{i}-\right.$ $\delta / m]$ for $m \geqslant 1$. Theorem 2.5 applied to the restriction of $f$ to $P_{m}, m \geqslant 1$, and Remark 2.8 yields the conclusion.

Let $I^{n}=\prod_{i=1}^{n}[0,1] \subset \mathbb{R}^{n}$ denote the unit $n$-cube. The group of dihomeomorphisms of $I^{n}$, denoted by $\operatorname{DiHomeo}\left(I^{n}\right)$, is a subgroup of the group of homeomorphisms of $I^{n}$. Let Homeo ${ }^{+}\left(I^{1}\right)$ denote the group of orientation preserving homeomorphisms of $I^{1}$.

Corollary 2.10. There is an isomorphism of topological groups:

$$
\left.\operatorname{DiHomeo}\left(I^{n}\right) \cong \operatorname{Homeo}^{+}\left(I^{1}\right)\right)^{n} \rtimes \Sigma_{n} \quad \text { (semidirect product) }
$$

where the symmetric group $\Sigma_{n}$ of permutations of $\underline{n}$ acts on $\left(\operatorname{Homeo}^{+}\left(I^{1}\right)\right)^{n}$ by permuting factors.

Proof. By Theorem 2.5,

$$
\operatorname{DiHomeo}\left(I^{n}\right)=\left\{\sigma_{*}\left(\prod_{i=1}^{n} q_{i}\right) \mid \sigma \in \Sigma_{n}, q_{i} \in \operatorname{Homeo}^{+}\left(I^{1}\right), i=1, \ldots, n\right\} .
$$

The map DiHomeo $\left(I^{n}\right) \rightarrow \Sigma_{n}$ given by $\sigma_{*}\left(\prod_{i=1}^{n} q_{i}\right) \mapsto \sigma$ is a group homomorphism, which is split by $\sigma \mapsto \sigma_{*}$, and its kernel is $\left(\operatorname{Homeo}^{+}\left(I^{1}\right)\right)^{n}$.

Let $B G$ denote the classifying space of the topological group $G$. Since Homeo $^{+}\left(I^{1}\right)$ is contractible, Corollary 2.10 yields the following result.

Corollary 2.11. BDiHomeo $\left(I^{n}\right)$ is homotopy equivalent to $B \Sigma_{n}$.

Remark 2.12. By Corollary 2.9, the corresponding versions of Corollaries 2.10 and 2.11 are valid for open cubes in place of closed cubes. 
Homology, Homotopy and Applications, vol.9(1), 2007

\section{References}

[1] S.D. Carson and P.F. Reynolds, The geometry of semaphore programs, ACM TOPLAS 9 (1987), 25-53.

[2] E.G. Coffman, M.J. Elphick and A. Shoshani, System deadlocks, Computing Surveys 3 (1971), 67-78.

[3] L.E. Fajstrup, E. Goubault and M. Raussen, Algebraic topology and concurrency, Theoret. Comput. Sci., 357 (2006), 241-278.

[4] P. Fernandes, Dihomotopy and concurrent computing, M.Sc. thesis, McMaster University, 2005.

[5] E. Goubault, Some geometric perspectives in concurrency theory, Algebraic topological methods in computer science (Stanford, CA, 2001), Homology, Homotopy Appl. 5 (2003), 95-136.

[6] M. Raussen, On the classification of dipaths in geometric models for concurrency. Math. Structures Comput. Sci. 10 (2000), 427-457.

[7] M. Raussen, State spaces and dipaths up to dihomotopy, Algebraic topological methods in computer science (Stanford, CA, 2001), Homology, Homotopy Appl. 5 (2003), 257-280.

[8] E.H. Spanier, Algebraic Topology, McGraw-Hill, New York, 1966.

Praphat Fernandes praphat.fernandes@emory.edu

Department of Mathematics and Computer Science

Emory University

Atlanta, Georgia 30322

USA

Andrew Nicas nicas@mcmaster.ca

Department of Mathematics and Statistics

McMaster University

Hamilton, Ontario L8S 4K1

Canada

This article is available at http://intlpress.com/HHA/v9/n1/a9 